\title{
Apr3 accelerates the senescence of human retinal pigment epithelial cells
}

\author{
SONG HAN, QINGJUN LU and NINGLI WANG \\ Beijing Tongren Eye Center, Beijing Ophthalmology \& Visual Sciences Key Laboratory, \\ Beijing Tongren Hospital, Capital Medical University, Beijing 100730, P.R. China
}

Received February 11, 2015; Accepted December 8, 2015

DOI: $10.3892 / \mathrm{mmr} .2016 .4926$

\begin{abstract}
Senescence of retinal pigment epithelium (RPE) cells is a major contributor to age-related macular degeneration (AMD). However, the molecular mechanisms underlying RPE dysfunction are not well understood. Apoptosis related protein 3 (Apr3) was originally cloned from HL-60 cells induced by all-trans retinoic acid (ATRA). Preliminary data revealed elevated Apr3 expression in the tissues of aged mice, suggesting that it is involved in the aging process. The present study demonstrated that Apr3 mRNA and protein levels were markedly increased in aged mouse RPE cells. Elevated Apr3 expression was also observed during premature senescence induced by oxidative stress $\left(\mathrm{H}_{2} \mathrm{O}_{2}\right.$ and tert-BHP) in ARPE-19 cells. Moreover, Apr3 overexpression promoted cellular senescence in ARPE-19 cells, as characterized by enhanced senescence-associated $\beta$-galactosidase activity, reduced cell proliferation and increased expression of the senescence markers p53 and p21. In addition, it was demonstrated that overexpression of Apr3-N, a truncated counterpart of Apr3, abrogated Apr3-induced phenotypes. It was concluded that Apr3 expression was induced in replicative and premature senescence of RPE cells and its overexpression accelerated senescence of ARPE-19 cells, which provides important insights into the function of Apr3 in senescence-associated diseases.
\end{abstract}

\section{Introduction}

Age-related macular degeneration (AMD) is the leading cause of irreversible blindness in developed countries and retinal pigment epithelium (RPE) is the primary affected tissue (1-3). Premature senescence has been implicated as

Correspondence to: Dr Ningli Wang, Beijing Tongren Eye Center, Beijing Ophthalmology \& Visual Sciences Key Laboratory, Beijing Tongren Hospital, Capital Medical University, 1 Dong Jiao Min Xiang, Beijing 100730, P.R. China

E-mail: ningliw63@163.com

Key words: apoptosis related protein 3, senescence, retinal pigment epithelium, oxidative stress, p53, p21 a potentially important pathophysiologic mediator of RPE dysfunction $(2,4-6)$. In vivo and in vitro studies have shown that primary human RPE from older patients or RPE cells exposed to oxidative stress exhibited senescence phenotypes, including hypertrophy, senescence-associated $\beta$-galactosidase (SA $\beta$-gal) activity, growth arrest and cell cycle arrest in the G1 phase $(3,4)$. However, the etiology and pathogenesis of AMD remain poorly understood.

Apoptosis related protein 3 (Apr3) was identified to be differentially expressed in HL-60 cells following treatment with all-trans retinoic acid (ATRA) compared with untreated cells as determined by a polymerase chain reaction (PCR)-based subtractive hybridization method (7). A previous study demonstrated that Apr3 overexpression arrested cells at the G1/S phase via inhibiting the transcriptional activity of Cyclin D1 (8). Zou et al (9) demonstrated that NELL-1 significantly inhibited osteoblast proliferation partly through interacting with Apr3, which resulted in the downregulation of Cyclin D1. However, the molecular mechanism by which Apr3 affects the cell cycle remains largely unknown. Based on previous studies, it was hypothesized that Apr3 may participate in cellular activities that are closely associated with the cell cycle, such as cell senescence, apoptosis and differentiation.

Our preliminary data revealed elevated Apr3 expression in the heart, lung, liver and kidney tissues of aged mice. To the best of our knowledge, the present study demonstrates for the first time that Apr3 levels were significantly increased in aged mouse RPE and prematurely senescent RPE cells induced by oxidative stress. Moreover, Apr3 overexpression in human RPE cells accelerated cellular senescence, which was abrogated by truncated Apr3. Thus, targeting Apr3 may represent a novel therapeutic strategy for delaying or inhibiting the progressive effects of senescence on AMD.

\section{Materials and methods}

Isolation of primary mouse RPE. All animal experiments were performed with the approval of the Institutional Animal Care and Use Committee at the Capital Medical University (Beijing, China). C57BL/6 mice were purchased from Capital Medical University. Mice of different ages (5 mice per group; age, 1, 6, 12 and 18 months) were maintained in a constant environment of $21 \pm 2^{\circ} \mathrm{C}$, with a humidity of $50 \pm 10 \%$ and a 12-h light/dark cycle. Food and water were available 
ad libitum. The mice were anesthetized with 2-3\% isoflurane (Halocarbon Products Corporation, Peachtree Corners, GA, USA). Mice were sacrificed by cervical dislocation and the eyes were enucleated and washed with Hanks' balanced salt solution (HBSS; Invitrogen; Thermo Fisher Scientific Inc., Waltham, MA, USA). The anterior segments were removed and the eyecups were incubated in $1 \mathrm{mg} / \mathrm{ml}$ hyaluronidase (Sigma-Aldrich, St. Louis, MO, USA) in HBSS for $1 \mathrm{~h}$ at $37^{\circ} \mathrm{C}$. The neural retina was peeled off and the RPE monolayers were cut into $2 \times 2 \mathrm{~mm}$ sections for RNA and protein extraction.

ARPE-19 cell culture. ARPE-19 represents a human RPE cell line that is widely used as a reproducible model of RPE cell biology and function. ARPE-19 cells were obtained from CoBioer Biosciences Co., Ltd. (Shanghai, China) and were routinely grown in F-12/Dulbecco's modified Eagle's medium (DMEM; 1:1, Invitrogen; Thermo Fisher Scientific Inc.) containing $10 \%$ fetal bovine serum (FBS; Invitrogen; Thermo Fisher Scientific Inc.), $2 \mathrm{mM}$ glutamine, $30 \mu \mathrm{g} / \mathrm{ml}$ penicillin and $50 \mu \mathrm{g} / \mathrm{ml}$ streptomycin (Sigma-Aldrich). ARPE-19 cells were passaged every 3-4 days.

Oxidative stress treatment. ARPE-19 cells were grown to 95\% confluence and were treated with various concentrations of $\mathrm{H}_{2} \mathrm{O}_{2}(0,25,50,100,150,200,250,300$ and $400 \mu \mathrm{M})$ diluted in ARPE medium for $2 \mathrm{~h}$. Then cells were washed with phosphate-buffered saline (PBS), and cultured in F12/DMEM for $22 \mathrm{~h}$. Similarly, ARPE-19 cells were treated with tert-butylhydroperoxide (tert-BHP, Sigma-Aldrich) at 0, 10, 20, 30, 40, 50, 100, 200 and $300 \mu \mathrm{M}$.

Lactose dehydrogenase ( $\mathrm{LDH}$ ) viability assay. ARPE-19 cells were seeded onto a 96-well microplate $\left(2 \times 10^{3}\right.$ cells/well). After $24 \mathrm{~h}$, cells were treated with various concentrations of $\mathrm{H}_{2} \mathrm{O}_{2}$ for $2 \mathrm{~h}$, washed once with PBS and cultured in normal medium for $22 \mathrm{~h}$. Cell viability was assessed by monitoring LDH release into the culture medium with an $\mathrm{LDH}$ cytotoxicity assay kit (Beyotime Institute of Biotechnology, Shanghai, China) according to the manufacturer's instructions. Cell viability was measured spectrophotometrically at $490 \mathrm{~nm}$ on a microplate reader (ELx800; Bio-Tek, Winooski, VT, USA). Data are presented as the mean \pm standard error of the mean of three replicates.

$\left[{ }^{3} \mathrm{H}\right]$-thymidine incorporation assay. ARPE-19 cells $\left(2 \times 10^{4}\right)$ were seeded onto 24 -well plates and underwent oxidative stress as described above. $\left[{ }^{3} \mathrm{H}\right]$-thymidine $(1 \mathrm{mCi} /$ well $)$ was added $4 \mathrm{~h}$ prior to cell harvesting and thymidine incorporation was measured by scintillation counting (PerkinElmer, Waltham, MA, USA).

Constructs. PcDNA3.1-hApr3 and pcDNA3.1-hApr3-N were provided by Dr Yu (Fourth Military Medical University, Xi'an, China) and subcloned into the pLVTHM-green fluorescent protein (GFP) lentivirus expression vector (Addgene, Inc., Cambridge,MA,USA) using Mlu1 and Cla 1 restriction enzymes (Takara Bio Inc., Otsu, Japan) to generate pLVTHM-hApr3 and pLVTHM-hApr3-N. 293FT cells were obtained from CoBioer Biosciences Co., Ltd) and maintained in DMEM containing $10 \%$ FBS, $2 \mathrm{mM}$ glutamine, $30 \mu \mathrm{g} / \mathrm{ml}$ penicillin and $50 \mu \mathrm{g} / \mathrm{ml}$ streptomycin (Sigma-Aldrich). pLVTHM-expressing plasmids with other helper vectors were co-transfected into 293FT cells and the lentiviruses were titrated by Cytomics FC 500 flow cytometry (Beckman Coulter, Inc., Brea, CA, USA). ARPE-19 cells $\left(2 \times 10^{6}\right)$ were seeded onto a $10-\mathrm{cm}$ plate and $100 \mu \mathrm{l}$ of Apr3-expressing lentiviruses were transduced. After 3 days, ARPE-19 cells were collected and GFP-positive cells were sorted by Cytomics FC 500 flow cytometry. The sorted cells were Apr3-overexpressing ARPE-19 stable cells and denoted as ARPE-Apr3.

SA $\beta$-gal activity assay. ARPE-Apr3 and the parent cells [ARPE-control (CTL)] were fixed in $4 \%$ paraformaldehyde (Beyotime Institute of Biotechnology) for $20 \mathrm{~min}$ and washed twice with PBS. Cells were then incubated overnight at $37^{\circ} \mathrm{C}$ with freshly prepared SA $\beta$-gal staining solution $(1 \mathrm{mg} / \mathrm{ml}$ $\mathrm{X}-\mathrm{Gal}, 40 \mathrm{mM}$ citric acid-sodium phosphate, $\mathrm{pH} 6.0 ; 5 \mathrm{mM}$ potassium ferrocyanide, $5 \mathrm{mM}$ potassium ferricyanide, $150 \mathrm{mM} \mathrm{NaCl}$ and $2 \mathrm{mM} \mathrm{MgCl}_{2}$ ). Cells were washed and visualized under a light microscope (CKX31; Olympus Corporation, Tokyo, Japan). The percentage of blue cells per 100 cells was calculated.

Reverse transcription (RT)-quantitative (q)PCR. Cells with indicated treatment were harvested for isolation of RNA using TRIzol reagent (Invitrogen, Thermo Fisher Scientific Inc.) according to the manufacturer's instructions. First-strand cDNA synthesis was performed using $2 \mu \mathrm{g}$ of RNA, random primers and catalyzed by M-MLV reverse transcriptase. qPCR was performed with the SYBR Premix Ex TaqTM (Takara Bio Inc.) containing DNA polymerase in $25 \mu \mathrm{l}$ reactions in 96-well PCR microplates (Applied Biosystems; Thermo Fisher Scientific, Inc.) using ABI PRISM 7500 Real-Time PCR system (Applied Biosystems; Thermo Fisher Scientific, Inc.) and StepOne software v2.0 (Applied Biosystems; Thermo Fisher Scientific, Inc.). PCR conditions were as follows: $95^{\circ} \mathrm{C}$ for $5 \mathrm{~min}$ followed by 35 cycles of $95^{\circ} \mathrm{C}$ for $20 \mathrm{sec}$ and $60^{\circ} \mathrm{C}$ for $20 \mathrm{sec}$. Primers sequences were as follows: Forward: $5^{\prime}$-tcagctgcagactctgatac- $3^{\prime}$ and reverse: 5'-gccagtaattgtcaacgaag-3' for Apr3; and forward: 5'-cgcggttctattttgttggt-3' and reverse: 5'-agtcggcatcgtttatggtc-3' for glyceraldehyde 3-phosphate dehydrogenase (GAPDH). Apr3 mRNA relative abundance was determined by normalizing to GAPDH using the $\Delta \mathrm{C}_{\mathrm{q}}$ method, where $\mathrm{C}_{\mathrm{q}}$ is the quantification cycle (10). The wells without a cDNA template were used as negative controls and GAPDH was used as a RT control. The experiment was repeated three times.

Western blot analysis. Cells were harvested and lysed in a radioimmunoprecipitation assay buffer (Thermo Fisher Scientific, Inc.) containing $50 \mathrm{mM}$ Tris-HC (pH 7.5), $150 \mathrm{mM}$ $\mathrm{NaCl}, 1 \% \mathrm{NP}-40,0.1 \%$ sodium dodecyl sulfate, $0.5 \%$ sodium deoxycholate and $50 \mathrm{mM} \mathrm{NaF}$. One tablet of protease inhibitor mixture (Complete Mini, Roche Applied Science, Indianapolis, IN, USA) was added prior to analysis. Protein concentration was measured using a Bradford reagent (Bio-Rad, Hercules, CA, USA). Then, $100 \mu \mathrm{g}$ protein lysates were separated by $12 \%$ SDS-polyacrylamide gel (Invitrogen; Thermo Fisher Scientific, Inc.) electrophoresis and transferred onto nitrocellulose membranes (Beyotime Institute of Biotechnology). Following blocking in a $5 \%$ non-fat dried milk solution in washing 

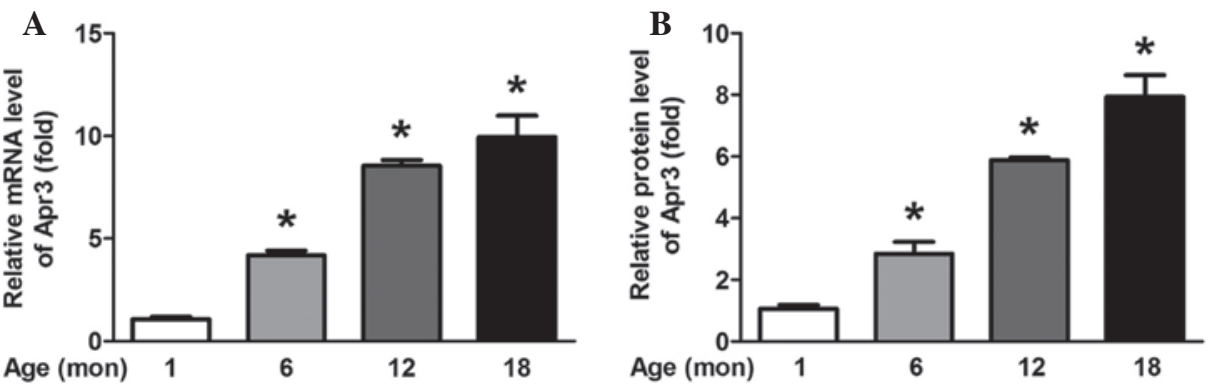

C

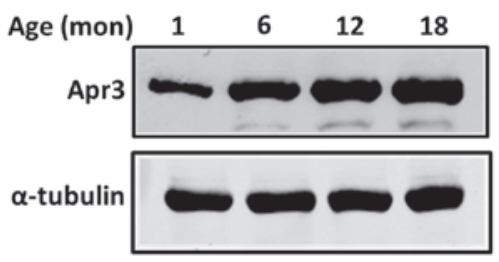

Figure 1. Elevated apoptosis related protein 3 (Apr3) expression in RPE cells from aged mice. RPE isolated from mice of different ages (1,6,12 and 18 months) was isolated and minced $(\mathrm{n}=6)$. (A) Reverse transcription-quantitative polymerase chain reaction was conducted to detect Apr3 mRNA levels. Values represent the mean \pm standard error of the mean. (B) The relative protein level of Apr3 was quantified. ${ }^{*} \mathrm{P}<0.05$ vs. the one-month-old mice. (C) Representative western blot of Apr3 from three different experiments. $\alpha$-tubulin served as an internal control.

A

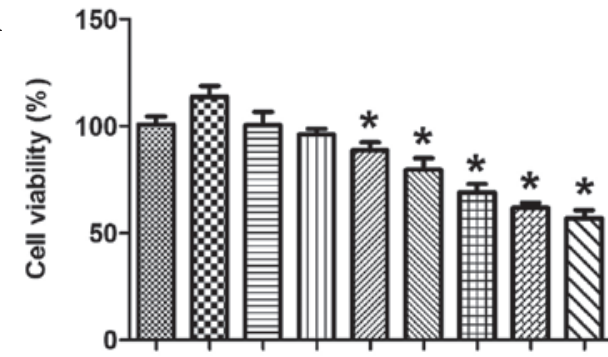

$\mathrm{H}_{2} \mathrm{O}_{2}(\mu \mathrm{M}) \quad 2^{\circ} \quad 5^{\circ}, 0^{\circ}, 5^{\circ} 2^{\circ} 2^{\circ} 5_{3} 0^{\circ} \times 0^{\circ}$

C

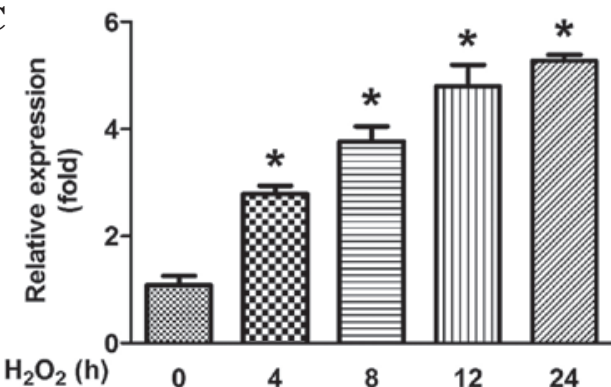

$\mathbf{E}$

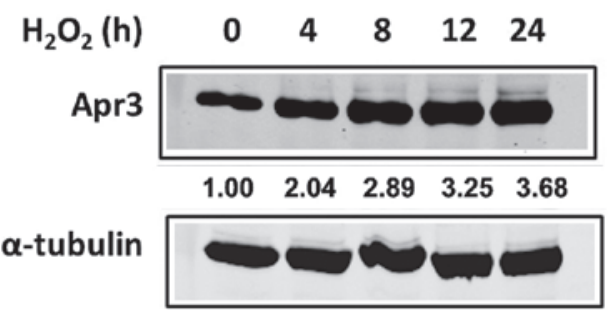

B

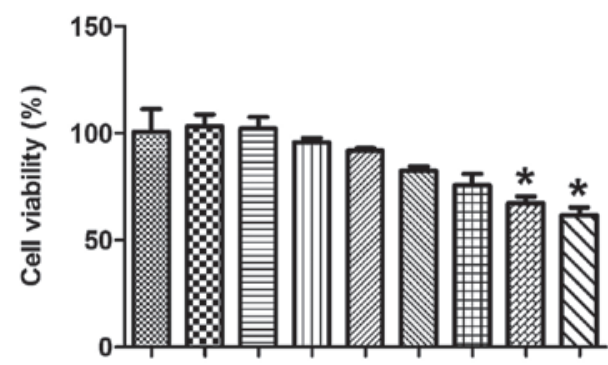

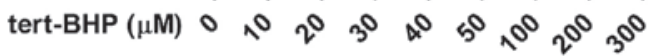

D

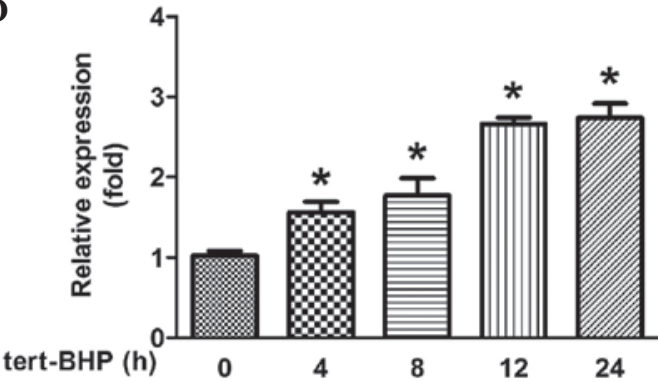

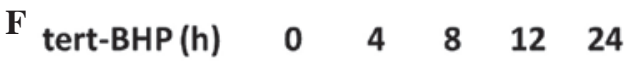

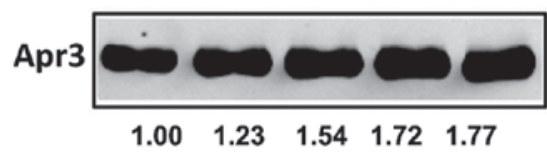

$\alpha$-tubulin

Figure 2. Increased apoptosis related protein 3 (Apr3) expression in oxidative stress induced-prematurely senescent ARPE-19 cells. ARPE-19 cells were treated with the indicated concentrations of (A) $\mathrm{H}_{2} \mathrm{O}_{2}$ and (B) tert-butylhydroperoxide (tert- $\mathrm{BHP}$ ) for $2 \mathrm{~h}$. Cell viability was determined by a lactose dehydrogenase assay after $22 \mathrm{~h}$. Values represent the mean \pm standard error of the mean of 6 wells from three independent experiments. (C and D) Reverse transcription-quantitative polymerase chain reaction was executed to detect Apr3 expression in ARPE-19 cells during oxidative stress-induced premature senescence at the indicated time point. The expression of Apr3 was normalized to glyceraldehyde 3-phosphate dehydrogenase and relative values were calculated using the level of Apr3 mRNA in the untreated cells as 1 . Values are represented as the mean \pm standard error of the mean. ${ }^{*}<0.05$ vs. the untreated cell group. (E and F) Apr3 protein levels were detected by western blot analysis. Results are representative of three different experiments.

buffer containing $10 \mathrm{mmol} / \mathrm{l}$ Tris $(\mathrm{pH} 7.5), 50 \mathrm{mmol} / \mathrm{l} \mathrm{NaCl}$ and $0.02 \%$ Tween 20 (TBST), membranes were incubated overnight at $4^{\circ} \mathrm{C}$ with rabbit polyclonal anti-Apr3 (1:2,000, Sigma-Aldrich; cat. no. SAB2100295), rabbit polyclonal anti- $\alpha$-tubulin $(1: 1,000$, Santa Cruz Biotechnology Inc., Dallas, TX, USA; cat. no. sc-5546) and two senescence markers, rabbit polyclonal anti-p21 (1:1,000; Santa Cruz Biotechnology, Inc.; cat. no. sc-756) and rabbit polyclonal anti-p53 (1:1,000; Santa Cruz Biotechnology, Inc.; cat. no. sc-6243). After washing three times with TBST, membranes were incubated for $1 \mathrm{~h}$ with goat anti-rabbit IgG horseradish peroxidase-coupled secondary antibodies (1:1,000, Santa Cruz Biotechnology Inc.; cat. no. sc-2004) at room temperature. Signals were detected with an enhanced chemiluminescence kit (Thermo 

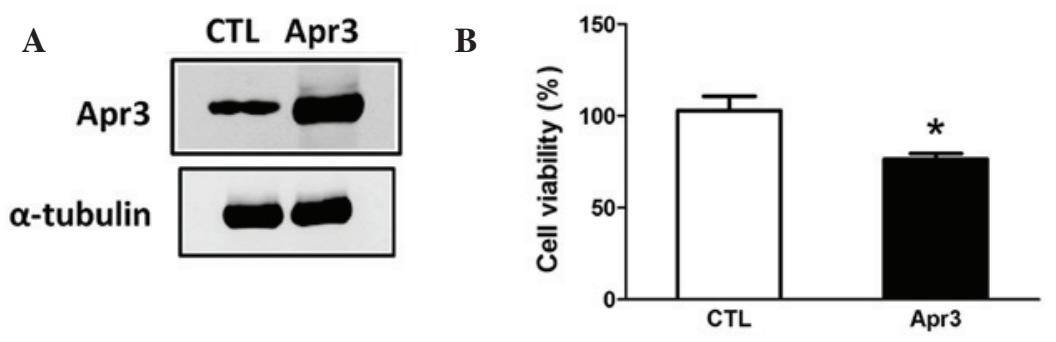

C

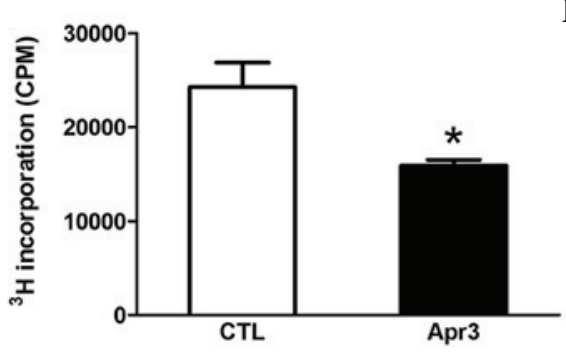

$\mathbf{E}$

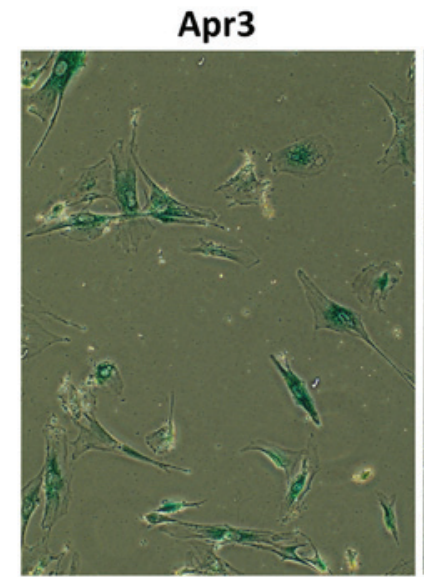

D
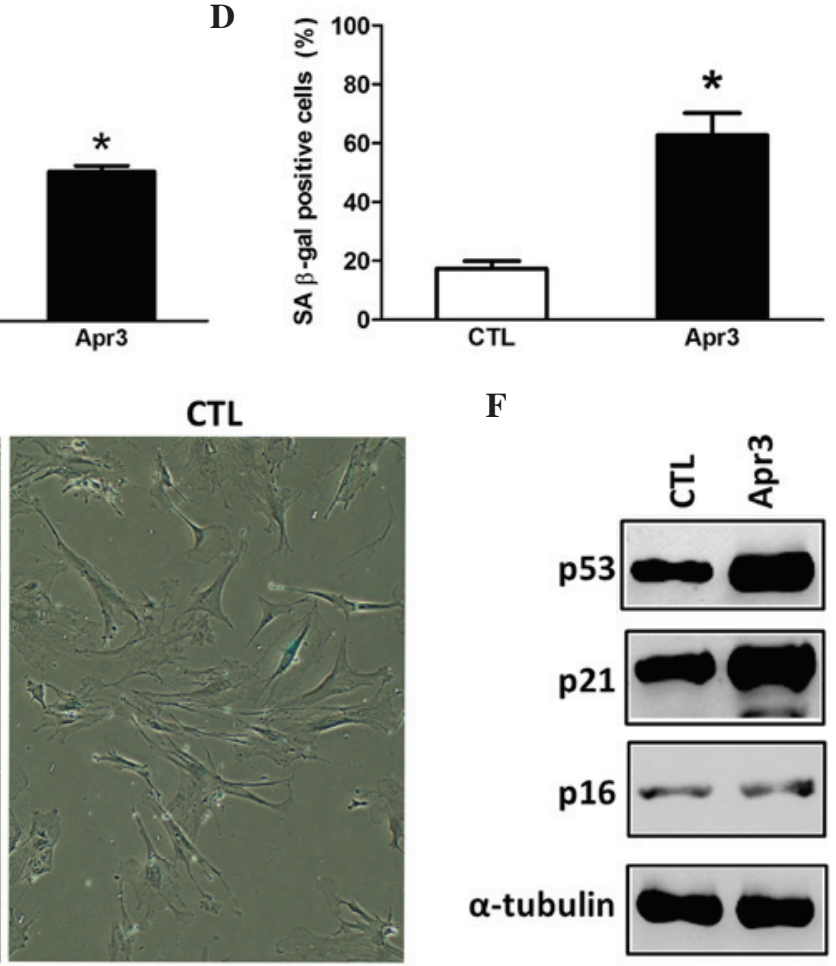

F

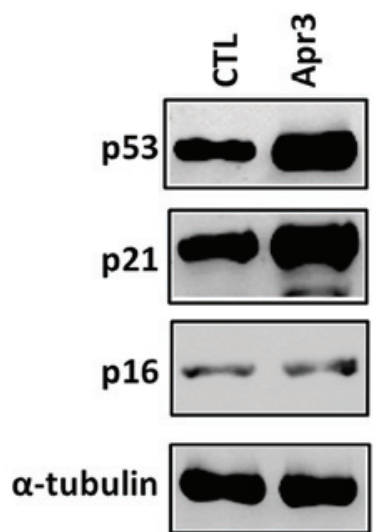

Figure 3. Accelerated senescence of ARPE-19 cells following apoptosis related protein 3 (Apr3) overexpression. (A) Apr3 expression was confirmed by western blot analysis in Apr3 overexpressing cells (APRE-Apr3) and parent cells (APRE-CTL). (B) APRE-Apr3 and APRE-CTL cells were seeded onto 24 -well plates. After $24 \mathrm{~h}$, cell viability was determined by a lactose dehydrogenase assay. Values are represented as the mean \pm standard error of the mean of 6 wells from three independent experiments. (C) APRE-Apr3 and APRE-CTL cells were seeded onto 24-well plates and cell proliferation was determined by $\left[{ }^{3} \mathrm{H}\right]$-thymidine incorporation assay. Values are represented as the mean \pm standard error of the mean of three independent experiments (D) The percentages of SA $\beta$-gal positive cells were plotted. Seven days after plating, SA $\beta$-gal positive cells were detected in APRE-Apr3 and APRE-CTL cells. Values are presented as the mean \pm standard error of the mean for three independent experiments. At least 100 cells were counted in each sample. (E) Representative images of stained cells are shown. Magnification, x40. (F) Representative western blots of p53, p21 and p16 in APRE-Apr3 and APRE-CTL cells 7 days after plating. ${ }^{*} \mathrm{P}<0.05$ vs. the CTL group. CTL, control.

Fisher Scientific Inc., Beijing, China). The scanned images were quantified using Kodak Digital Science one-dimensional software (Eastman Kodak Co., New Haven, CT, USA).

Statistical analysis. Data are presented as the mean \pm standard error of the mean. Unpaired Student's t-test was used for statistical analysis with GraphPad Prism version 5.02 (GraphPad Software, Inc., La Jolla, CA, USA). P $<0.05$ was considered to indicate a statistically significant difference.

\section{Results}

Elevated Apr3 level in the RPE from aged mice. Our preliminary data showed a significant increase in the Apr3 expression level in aged mouse tissues, including heart, lung, liver and kidney tissue (data not shown), which suggested that Apr3 may function in the aging process. Current evidence indicates that senescent RPE cells accumulate with age in vivo $(4,5,11)$. In order to assess whether Apr3 exhibits a role in RPE cell senescence, the endogenous expression of Apr3 was detected in RPE cells from young and aged mice. As expected, the mRNA level of Apr3 gradually increased with age (Fig. 1A). Consistently, the Apr3 protein level in RPE cells showed a similar trend and its level in 18-month-old mice was enhanced by 7.94 -fold compared with that in one-month-old mice (Fig. 1B and C).

Apr3 is upregulated in oxidative stress-induced senescent human ARPE-19 cells. In AMD, chronic low level oxidative stress is hypothesized to accelerate RPE cell senescence, thus promoting disease pathogenesis $(4,5)$. The present study examined whether Apr3 expression was induced in premature senescence caused by oxidative stress in ARPE-19 cells (a non-transformed human RPE cell line). Initially, culture conditions that induced ARPE-19 senescence without causing 
A

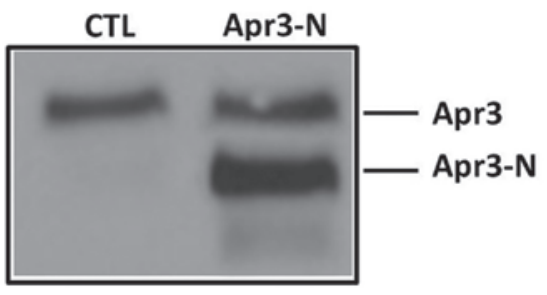

B

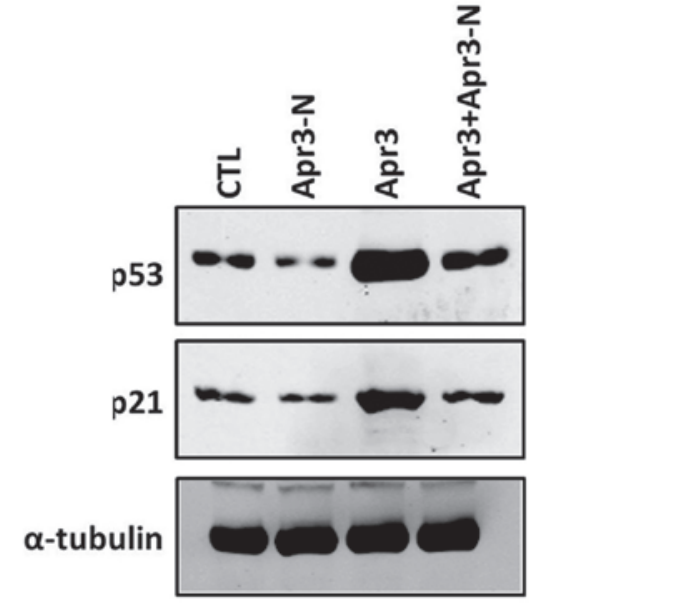

C

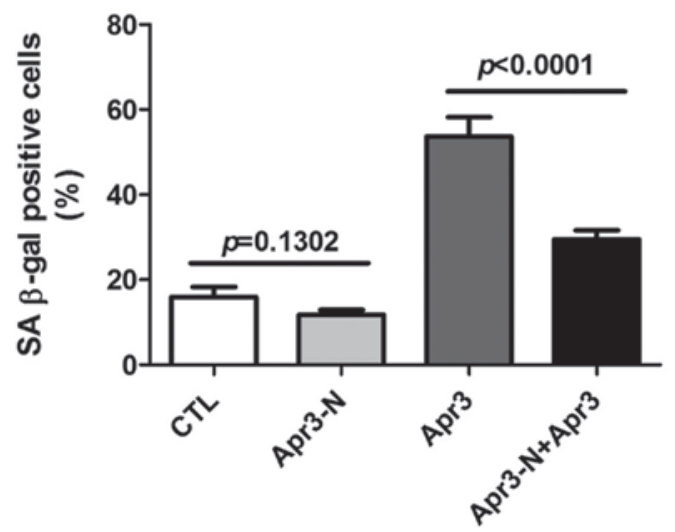

Figure 4. Apoptosis related protein 3 (Apr3)-induced phenotype is abrogated by Apr3-N. (A) ARPE-19 cells were transduced with Apr3-N-overexpressing lentivirus (Apr3-N) and its protein level was determined by western blot analysis. Endogenous (Apr3) and truncated (Apr3-N) Apr3 were indicated (B) Seven days after transduction, the percentage of senescence-associated $\beta$-galactosidase (SA $\beta$-gal) positive cells were determined. Values are presented as the mean \pm standard error of the mean for three independent experiments. At least 100 cells were counted for each sample. (C) Western blot analysis was executed to detect p53 and p21 levels in the cells. $\alpha$-tubulin was used as an internal control. Results are representative of three different experiments

cell death were investigated using an LDH activity assay. ARPE-19 cells were treated with various concentrations of $\mathrm{H}_{2} \mathrm{O}_{2}$ for $2 \mathrm{~h}$ and their viability was assayed after $22 \mathrm{~h}$. It was demonstrated that $\mathrm{H}_{2} \mathrm{O}_{2}$ at $\leq 100 \mu \mathrm{M}$ was not cytotoxic, whereas concentrations of $\geq 150 \mu \mathrm{M}$ were (Fig. 2A). Similarly, different concentrations of tert-BHP were applied. It was demonstrated that tert-BHP at $\leq 40 \mu \mathrm{M}$ was not cytotoxic (Fig. 2B). Thus, $\mathrm{H}_{2} \mathrm{O}_{2}$ at $100 \mu \mathrm{M}$ and tert-BHP at $40 \mu \mathrm{M}$ were selected as the optimal oxidative stress doses to treat RPE cells in the following experiments. As expected, Apr3 mRNA levels were enhanced in a time-dependent manner following treatment with $\mathrm{H}_{2} \mathrm{O}_{2}$ and tert-BHP (Fig. 2C and D). Consistently, $\mathrm{H}_{2} \mathrm{O}_{2}$ and tert-BHP resulted in a 3.68- and 1.77-fold increase in Apr3 protein levels following $24 \mathrm{~h}$ of treatment, respectively (Fig. $2 \mathrm{E}$ and $\mathrm{F}$ ). Thus, the results suggest that elevated endogenous Apr3 expression may be involved in RPE cell senescence.

Apr3 overexpression accelerates senescence of ARPE-19 cells. To investigate the putative role of Apr3 in cell senescence, Apr3-expressing lentivirus was engineered and transfected into ARPE-19 cells. Subsequently, stably elevated Apr3 protein levels were confirmed in ARPE-19 cells (Fig. 3A). Cell viability was then determined using an LDH activity assay and it was demonstrated cells overexpressing Apr3 exhibited decreased cell viability compared with the parent cells (Fig. 3B, 76.4 vs. 101.9\%). Cellular senescence is a stress-response phenomenon where cells lose the ability to proliferate $(2,3)$. Thus, it was then assessed whether Apr3 influenced the proliferative properties of ARPE-19 cells. As shown in Fig. 3C, Apr3 overexpression moderately inhibited cell proliferation by $34.5 \%$ compared with the parent cells as determined by a ${ }^{3}[\mathrm{H}]$-thymidine incorporation assay.

Following 7 days of culture, SA $\beta$-gal staining demonstrated that $\sim 62.7 \%$ of cells overexpressing Apr3 were positive for SA $\beta$-gal, compared with $17.3 \%$ of the parent cells (Fig. 3D and $\mathrm{E})$. The expression levels of senescence markers p21 and p53 in Apr3 overexpressed cells were elevated by 4.3- and 3.5-fold compared with the parent cells, respectively (Fig. 3F). However, p16 level was barely altered upon Apr3 overexpression. The above data demonstrated that Apr3 overexpression produced an accelerated prematurely senescent phenotype in ARPE-19 cells.

Truncated Apr3 abolished Apr3-induced senescence. A previous study showed that Apr3-N, a truncated counterpart of Apr3, strongly antagonized Apr3-induced cell cycle arrest (8). To further confirm Apr3-caused senescent phenotype, Apr3-N lentivirus was transduced into cells overexpressing Apr3 and parent cells (Fig. 4A), and cell senescence was evaluated. Fig. 4B showed that Apr3-N overexpression in parent cells resulted in a marginal decrease of SA $\beta$-gal positive cells compared with parent cells (15.9 vs. $12.3 \%, \mathrm{P}=0.1302$ ). However, in Apr3 overexpressing cells, the percentage of SA $\beta$-gal positive cells was markedly decreased from 53.7 to 29.5\% following Apr3-N overexpression (Fig. 4B, P<0.0001). Although Apr3-N overexpression alone inhibited p21 and p53 expression in parent cells (lane 2 vs. lane 1), both protein levels were markedly decreased by Apr3-N plus Apr3 overexpression (Fig. 4C, lane 4 vs. lane 3). Altogether, the data indicated that Apr3-N could abrogate the Apr3-induced cellular senescence phenotype.

\section{Discussion}

A previous study demonstrated that Apr3 overexpression arrested the cell cycle at the G1/S phase by inhibiting Cyclin D1 transcription (8). However, it remains to be determined whether Apr3 affects cell behaviors other than cell proliferation. To the best of our knowledge, the present study provided the first evidence that the Apr3 level was increased in aged mouse RPE cells and that oxidative stress-induced prematurely senescent ARPE-19 cells, as well as the fact that Apr3 overexpression 
markedly promoted ARPE-19 senescence. Further investigation is required to explore the molecular mechanisms responsible for the Apr3-induced senescent phenotype.

Bioinformatics analysis demonstrated that Apr3 protein shares $46 \%$ homology with plasma membrane-localized Notch ligands (unpublished data). Yu et al (8) showed that Apr3 was a membrane protein in breast cancer cells. Kammula et al (12) identified that Apr3 was a novel membrane-localized interaction partner of Nef. Therefore, Apr3 may function as a membrane protein to transduce extracellular signals into cells. This hypothesis was partially supported by a study by $\mathrm{Yu}$ et al (8), which demonstrated that Apr-N, truncated Apr3 with deletion of its intracellular region, displayed classical cellular distribution of secretory proteins and markedly antagonized Apr3 induced cell cycle arrest (8). Similarly, it was demonstrated that ARPE-19 cells overexpressing Apr3-N exhibited a moderate decrease in the level of senescence compared with cells overexpressing Apr3, and Apr3-N overexpression abrogated Apr3-induced senescence of ARPE-19 cells. Since Apr3-N overexpression did not alter the endogenous Apr3 level, it was hypothesized that it may neutralize putative extracellular proteins that interact with Apr3 or interfere with the interaction between Apr3 and its partners, thus partially or completely blocking Apr3-mediated signal transduction and its subsequent function. Therefore, it will be interesting to examine whether Apr3 is localized in the membrane of RPE cells, or whether Apr3 translocates between the cell membrane and the cytoplasm during senescence.

Dysregulation of growth factor expression in RPE cells has been implicated as an important pathological mechanism in $\operatorname{AMD}(1,5)$. Increased expression of vascular endothelial growth factor (VEGF) and pigment epithelial-derived factor (PEDF) by RPE were identified in AMD and are effective therapeutic targets for $\operatorname{AMD}(3,13,14)$. However, little is known regarding the growth factor microenvironment mediating pathological changes in AMD (4). Accumulating evidence demonstrates that ATRA markedly increased VEGF and PEDF expression, and ATRA emerged as the most potent inducer among the various RAs $(15,16)$. Apr3 levels were markedly increased in ATRA-treated HL-60 cells (7), therefore, it was hypothesized that ATRA may regulate Apr3 expression in RPE cells and result in the development of AMD.

Several signal transduction pathways are involved in the process of cellular senescence, including p53/p21 pathway, p16/Rb pathway and insulin/insulin-like growth factor-1 (IGF1) signal pathway (17-19). In the present study, it was demonstrated that Apr3 induced increases in p53 and p21 expression, whereas the p16 level was not altered. Collectively, it was hypothesized that the Apr3-induced phenotype may be partly achieved through the p53/p21 signaling pathway. Elucidating the molecular mechanism by which Apr3 acts on senescence may be beneficial for the understanding of its function.

In conclusion, the results from the present study demonstrate, for the first time, that Apr3 overexpression in human RPE cells accelerated cellular senescence. The role of cellular senescence in a variety of age-associated pathologies is becoming increasingly accepted. Current findings may aid in investigating the function of Apr3 in other senescence-associated diseases, such as cancer, Alzheimer's disease, muscle atrophy and cardiovascular disease.

\section{Acknowledgements}

The authors would like to thank Dr Fang Yu at the Fourth Military Medical University (Xi'an, China) for providing the pcDNA3.1-hApr3 and pcDNA3.1-hApr3-N vectors.

\section{References}

1. de Jong PT: Age-related macular degeneration. N Engl J Med 355: 1474-1485, 2006.

2. Plafker SM, O'Mealey GB and Szweda LI: Mechanisms for countering oxidative stress and damage in retinal pigment epithelium. Int Rev Cell Mol Biol 298: 135-177, 2012.

3. Makarev E, Cantor C, Zhavoronkov A, Buzdin A, Aliper A and Csoka AB: Pathway activation profiling reveals new insights into age-related macular degeneration and provides avenues for therapeutic interventions. Aging (Albany NY) 6: 1064-1075, 2014.

4. Cao S, Walker GB, Wang X, Cui JZ and Matsubara JA: Altered cytokine profiles of human retinal pigment epithelium: Oxidant injury and replicative senescence. Mol Vis 19: 718-728, 2013.

5. Sharma K, Sharma NK and Anand A: Why AMD is a disease of ageing and not of development: Mechanisms and insights. Front Aging Neurosci 6: 151, 2014.

6. Hjelmeland LM, Cristofolo VJ, Funk W, Rakoczy E and Katz ML: Senescence of the retinal pigment epithelium. Mol Vis 5: 33, 1999.

7. Zhu F, Yan W, Zhao ZL, Chai YB, Lu F, Wang Q, Peng WD, Yang AG and Wang CJ: Improved PCR-based subtractive hybridization strategy for cloning differentially expressed genes. Biotechniques 29: 310-313, 2000.

8. Yu F, Yang G, Zhao Z, Ji L, Cao Y, Bai L, Lu F, Fu H, Huang B, $\mathrm{Li} \mathrm{H}$, et al: Apoptosis related protein 3, an ATRA-upregulated membrane protein arrests the cell cycle at G1/S phase by decreasing the expression of cyclin D1. Biochem Biophys Res Commun 358: 1041-1046, 2007.

9. Zou X, Shen J, Chen F, Ting K, Zheng Z, Pang S, Zara JN, Adams JS, Soo C and Zhang X: NELL-1 binds to APR3 affecting human osteoblast proliferation and differentiation. FEBS Lett 585: 2410-2418, 2011.

10. Livak KJ and Schmittgen TD: Analysis of relative gene expression data using real-time quantitative PCR and the 2(-Delta Delta C(T)) Method. Methods 25: 402-408, 2001.

11. Jeyapalan JC, Ferreira M, Sedivy JM and Herbig U: Accumulation of senescent cells in mitotic tissue of aging primates. Mech Ageing Dev 128: 36-44, 2007.

12. Kammula EC, Mötter J, Gorgels A, Jonas E, Hoffmann S and Willbold D: Brain transcriptome-wide screen for HIV-1 Nef protein interaction partners reveals various membrane-associated proteins. PLoS One 7: e51578, 2012.

13. He Y, Leung KW, Ren Y, Pei J, Ge J and Tombran-Tink J: PEDF improves mitochondrial function in RPE cells during oxidative stress. Invest Ophthalmol Vis Sci 55: 6742-6755, 2014.

14. Ablonczy Z, Dahrouj M and Marneros AG: Progressive dysfunction of the retinal pigment epithelium and retina due to increased VEGF-A levels. FASEB J 28: 2369-2379, 2014.

15. Tombran-Tink J, Lara N, Apricio SE, Potluri P, Gee S, Ma JX, Chader $\mathrm{G}$ and Barnstable CJ: Retinoic acid and dexamethasone regulate the expression of PEDF in retinal and endothelial cells. Exp Eye Res 78: 945-955, 2004.

16. Chen JT, Liang JB, Chou CL, Shyu RC and Lu DW: Retinoic acid induces VEGF gene expression in human retinal pigment epithelial cells (ARPE-19). J Ocul Pharmacol Ther 21: 413-419, 2005.

17. Bartke A: Impact of reduced insulin-like growth factor-1/insulin signaling on aging in mammals: Novel findings. Aging Cell 7: 285-290, 2008

18. Dvashi Z, Green Y and Pollack A: TAK1 inhibition accelerates cellular senescence of retinal pigment epithelial cells. Invest Ophthalmol Vis Sci 55: 5679-5686, 2014.

19. Salama R, Sadaie M, Hoare M and Narita M: Cellular senescence and its effector programs. Genes Dev 28: 99-114, 2014. 